\title{
Digestibilité in vitro et dégradabilité in situ dans le rumen de ligneux fourragers disponibles sur pâturages S. T. Fall " naturels au Sénégal. Premiers résultats
}

\begin{abstract}
FALL (S.T.). Digestibilité in vitro et dégradabilité in situ dans le rumen de ligneux fourragers disponibles sur pâturages naturels au Sénégal. Premiers résultats. Revue Elev. Méd. vét. Pays trop., 1991, 44 (3) : 345354

Pour évaluer l'utilisation digestive des ligneux fourragers disponibles sur pâturages naturels des zones soudanienne et sahélienne du Sénégal, leur digestibilité in vitro (méthode de Tilley-Terry) et leur dégradabilité dans le rumen (méthode in situ) sont déterminées. Sur 58 échantillons analysés in vitro, la digestibilité in vitro de la matière sèche est en moyenne de 51 avec des extrêmes de 88 à $26 \mathrm{p}$. 100. La digestihilité in vitro de la matière organique a varié de 84 à 15 avec une moyenne de 42 p. 100. Trente-huit profils de dégradation dans le rumen sont réalisés sur 24 espèces. Les dégradabilités moyennes des matières azotées totales et de la matière sèche sont respectivement de 59 et 57 p. 100. Des facteurs de variation liés à l'espèce, l'organe et l'âge ont été observés. Une hiérarchie provisoire des espèces étudiées est proposée. Mots clés : Plante ligneuse - Fourrage - Ruminant Digestibilité in vitro - Dégradabilité in situ - Sénégal.
\end{abstract}

\section{INTRODUCTION}

Les ligneux représentent une part importante de la biomasse fourragère disponible au Sahel. Supplément protéique pour les bovins, arbres et arbustes fourragers peuvent constituer la base de l'alimentation des petits ruminants pendant une grande partie de la saison sèche. Leur principal critère de valeur est la teneur en matières azotées qui peut atteindre 35 p. 100 de la matière sèche, avec des valeurs moyennes proches de $15 \mathrm{p}$. 100 . Ils sont donc plus riches en matières azotées que les herbacées dont la teneur décroît de 15-20 p. 100 de la matière sèche en début de cycle à des valeurs inférieures à 5 p. 100 au stade "pailles sur pied", qui constitue l'essentiel de la biomasse en milieu et fin de saison sèche.

De nombreux travaux ont déjà porté sur la composition chimique des espèces ligneuses consommées en milieu tropical $(2,7,11,13)$. Il existe cependant peu de données sur leur utilisation digestive, tant pour la disponibilité de la matière organique que des matières azotées. On sait que les teneurs en lignine peuvent être importantes et jouer un rôle inhibiteur dans la disponibilité de l'azote et de la matière organique ; les teneurs en constituants pariétaux

1. Institut Sénégalais de Recherches Agricoles (ISRA), Laboratoire National d'Élevage et de Recherches Vétérinaires (LNERV), BP 2057, Dakar, Sénégal.

* Avec la collaboration technique de B. DIAW et W. GOUDIABY.

Reçu le 13.12.1989, accepté le 24.9.1991. diffèrent suivant l'organe et le stade phénologique ; mais les facteurs de variation sont cependant peu connus. De même, l'influence négative des tannins sur la valeur nutritive des ligneux est souvent citée $(2,14,16,20)$.

Ce travail a pour but d'évaluer la digestibilité in vitro et la dégradabilité dans le rumen de ligneux fourragers des zones sahélienne et soudano-sahélienne du Sénégal. Les résultats devraient contribuer à identifier les espèces ayant les meilleures valeurs nutritives et, pour certaines d'entre elles, à décrire leurs variations saisonnières. Les essais ont été réalisés de 1987 à 1989 au LNERV-ISRA de Dakar (Sénégal).

\section{MATÉRIEL ET MÉTHODE}

\section{Échantillons}

Iis ont été récoltés dans le Ferlo (zone sahélienne) et le Sine-Saloum (zone sahélo-soudanienne) (tabl. I et II) puis séchés au soleil et passés au broyeur à marteau équipé d'une grille de $1 \mathrm{~mm}$. Les échantillons analysés in vitro et ceux étudiés in situ ne sont pas les mêmes, mais les résultats obtenus ont été comparés pour confronter les hiérarchies des espèces établies par les deux techniques.

\section{Digestibilite in vitro}

La méthode en deux temps de TILLEY et TERRY (19) a été appliquée pour évaluer la digestibilité de 58 échantillons. Le jus de rumen a été prélevé sur trois taurillons fistulés du rumen et d'un poids moyen de $250 \mathrm{~kg}$. Ils recevaient de la paille de riz à volonté, $1 \mathrm{~kg}$ de tourteau d'arachide et $75 \mathrm{~g}$ de poudre d'os en deux repas par jour ( $8 \mathrm{~h}$ et $14 \mathrm{~h}$ ). La prise d'essai de $0,5 \mathrm{~g}$ d'échantillon subit deux traitements de $48 \mathrm{~h}$ chacun. La première incubation est réalisée dans un mélange de salive artificielle et de jus de rumen filtré. On procède ensuite à une attaque enzymatique par une solution de pepsine acidifiée, puis à la filtration, sous vide, avec un creuset (Alundun $n^{\circ} 3$ ) préalablement taré. Après dessiccation puis calcination, les pourcentages de matière sèche et de matière organique disparus représentent, respectivement, la digestibilité in vitro de la matière sèche (DIVMS) et celle de la matière organique (DIVMO). Chaque échantillon est incubé en 


\section{S. T. Fall}

TABLEAU I Digestibilité in vitro des ligneux. Premiers résultats. Valeurs moyennes (extrêmés).

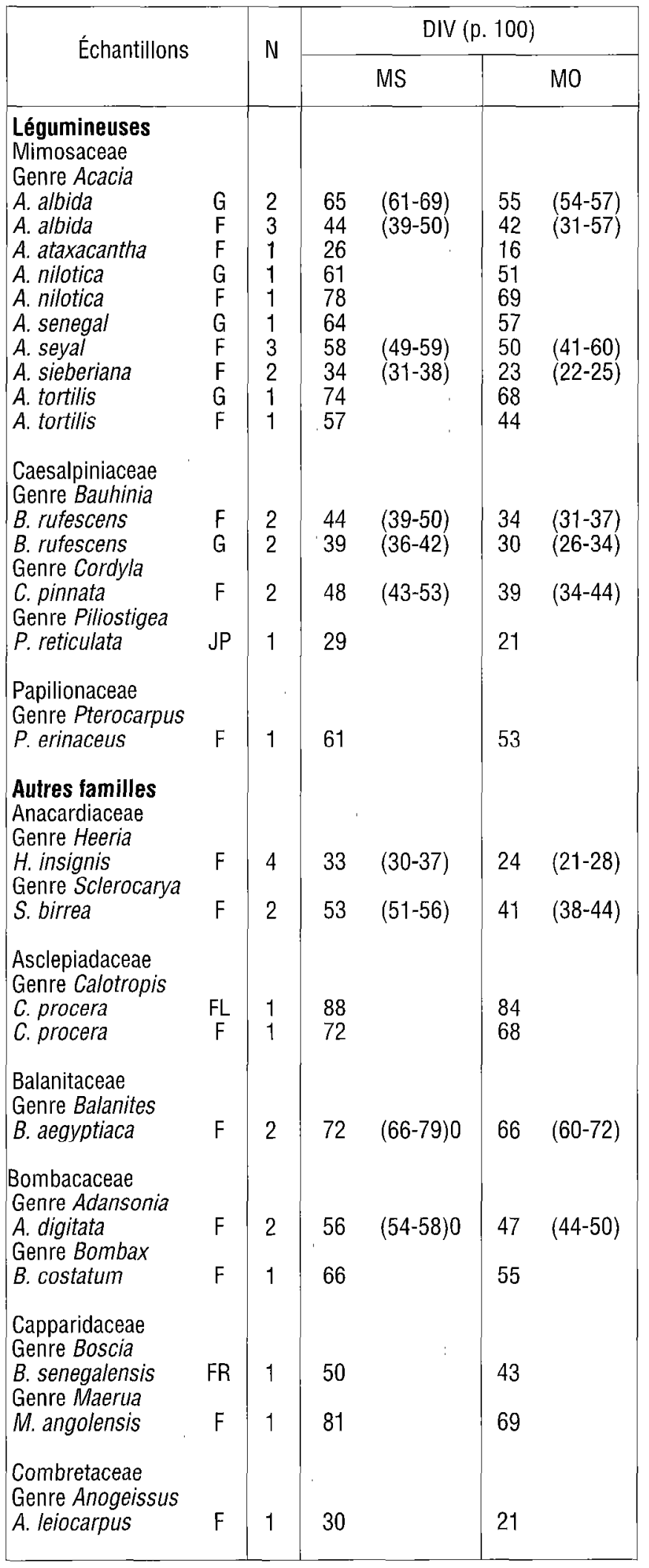

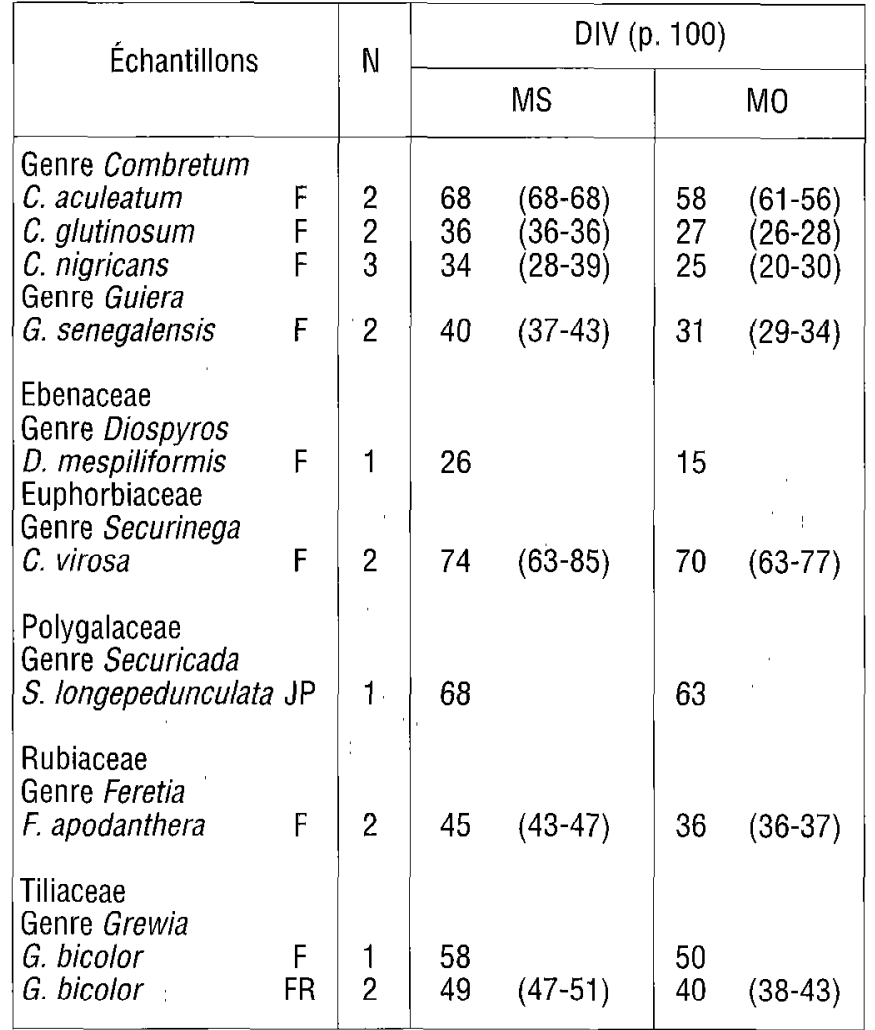

$F$ : feuilles ; $F L$ : fleurs : FR : fruits ; $G$ : gousses de légumineuses ; $E$ : écorces; JP : jeunes pousses (= jeunes rameaux, feuilles). 
TABLEAU II Dégradabilité in situ de la matière sèche des ligneux. Premiers résultats.

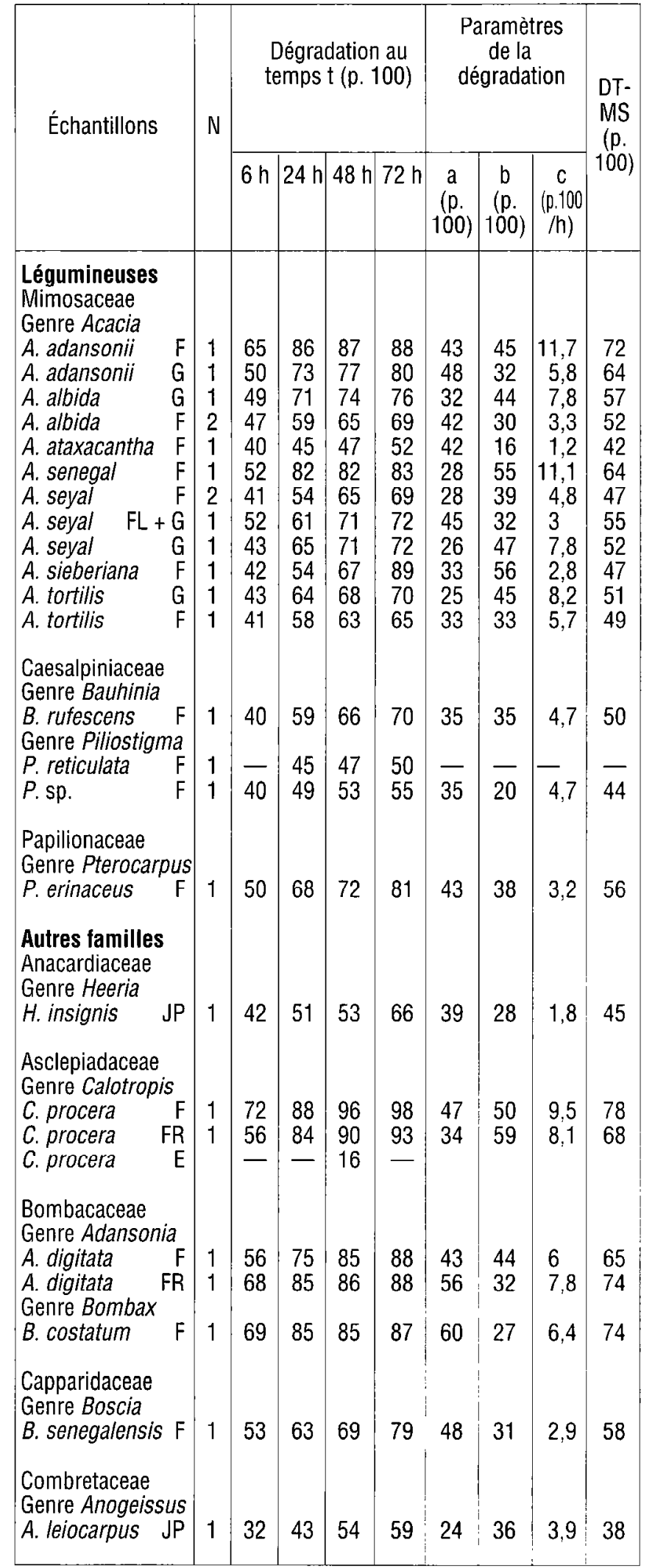

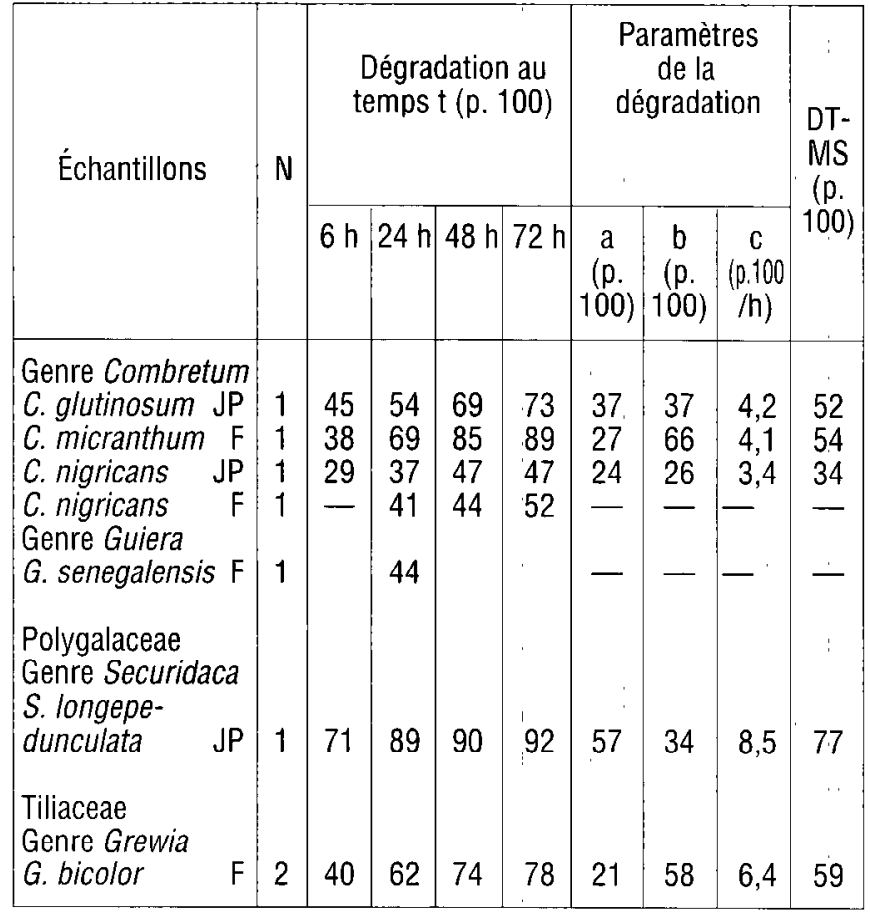

$F$ : feuilles ; $F L$ : fleurs ; FR : fruits ; $G$ : gousses de légumineuses; $E$ : écorces; JP : jeunes pousses (= jeunes rameaux, feuilles). 
triple et l'essai répété à une semaine d'intervalle ; il y a donc six répétitions par mesure. Un témoin de digestibilité connue est introduit dans chaque série pour le contrôle.

\section{Dégradabilité in situ dans le rumen}

$$
(3,4,15)
$$

Les animaux sont les mêmes que ceux utilisés précédemment dans la méthode de Tilley-Terry.

Cinq grammes d'échantillon broyé sont introduits dans un sachet de nylon (10-15 cm, porosité de 46 microns, tissu Blutex, Tripette et Renaud) scellé à la chaleur. Six sachets par échantillon sont placés dans le rumen de chaque taurillon une demi-heure après le premier repas du matin. Apres 3, 6, 9, 24, 48 et 72 heures d'incubation, ils sont retirés et lavés par massage sous le robinet jusqu'à ce que l'eau soit claire. Ils sont ensuite séchés à l'étuve à $80^{\circ} \mathrm{C}$. Un temps zéro est réalisé par lavage à l'eau d'un sachet contenant une prise d'essai. Deux mesures ont été réalisées sur trois bovins, soit six répétitions par aliment et temps d'incubation.

Les teneurs en matière sèche (dessiccation à l'étuve à $80^{\circ} \mathrm{C}$ ) et en matières azotées totales (méthode de Kjedahl) ont été déterminées sur le substrat et le résidu.

La dégradabilité (DT) des aliments est fonction de leur vitesse de dégradation mesurée in situ dans le rumen et de la vitesse de transit (k) des petites particules qui est estimée à 6 p. 100/h (15). La cinétique de dégradation a été appréciée par un ajustement exponentiel exprimé par l'équation :

$$
D=a+b\left(1-e^{-c t}\right)
$$

où $D(p .100)$ représente la dégradation mesurée au temps $t, a(p .100)$ la fraction immédiatement dégradable, b (p. 100) la fraction dégradable à une vitesse $c$ (p. 100/h). La dégradabilité théorique (DT) est calculée par la relation:

$\mathrm{DT}=\mathrm{a}+(\mathrm{bc}) /(\mathrm{c}+\mathrm{k}), \mathrm{k}$ représentant le turnover des particules dans le rumen, soit 6 p. 100/h.

Les ajustements ont été effectués pour la matière sèche et les matières azotées avec une régression non linéaire du logiciel STAT-ITCF (18).

\section{RÉSULTATS}

\section{Digestibilité in vitro}

Cinquante-huit échantillons provenant de différents organes de trente espèces d'arbres ou d'arbustes fourragers ont fait l'objet de mesures (tabl. I). Les résultats sont très variables d'une espèce ligneuse à une autre. La DIVMS varie de 26 à 88 p. 100 , la moyenne étant de 51 p. 100.

La DIVMO et la DIVMS varient dans le même sens. Les deux paramètres sont bien corrélés ( $r=0,97$, erreur standard $4,8 p$. $100, n=58$ ). L'écart moyen entre les deux valeurs est de dix points de digestibilité.

Les résultats sont présentés par famille, espèce et organe (tabl. l). Des différences apparaissent entre groupes et à l'intérieur d'une espèce selon l'organe et l'âge de celuici. Toutefois, au regard des 600 espèces ligneuses identifiées dans la zone soudano-sahélienne, les effectifs étudiés ici ne permettent pas de procéder à des comparaisons entre familles et genres, l'effectif le plus élevé par famille étant de sept espèces (Mimosaceae). Le classement qui peut être fait d'après les tableaux n'est donc pas définitif, ni généralisable. Ces données sont avant tout destinées à illustrer les variations de digestibilité des ligneux. Certains résultats méritent cependant d'être soulignés, en particulier ceux relatifs à des familles, des espèces ou des organes échantillonnés à plusieurs reprises.

Avec une DIVMO moyenne supérieure à 70 p. 100, les organes des espèces appartenant aux familles des Asclepiadaceae, Balanitaceae et Euphorbiaceae sont les plus digestibles. Pour les familles des Mimosaceae (à l'exception d'A. ataxacantha et d'A. sieberiana), Papilionaceae, Bombacaceae, Capparidaceae, Polygalaceae et Tiliaceae, la DIVMO comprise entre 45 et 63 p. 100 est intermédiaire tandis que celle des Anacardiaceae, Caesalpiniaceae, Combretaceae (à l'exception de C. aculeatum) et Rubiaceae, variant de 32 à 36 p. 100, est médiocre.

Parmi les 16 échantillons de feuilles de légumineuses représentant 11 espèces, quatre ont eu des DIVMO très faibles, proches de 30 p. 100 (Acacia ataxacantha, A. sieberiana, Piliostigma reticulata), cinq ont eu des DIVMO faibles, entre 30 et 50 p. 100 (Acacia albida 1 sur 2, $A$. seyal 1 sur 3, A. tortilis, Bauhinia rufescens, Cordyla pinnata), et sept ont eu des DIVMO moyennes ou fortes, supérieures à 50 p. 100 (Acacia albida 1 sur 2 , A. seyal 2 sur 3, A. nilotica, $A$. senegal, Pterocarpus erinaceus).

Les feuilles de certaines espèces récoltées à différentes périodes mettent en évidence des variations saisonnières. Ainsi, les feuilles d'Acacia albida récoltées en janvier sont plus digestibles que celles de juin (DIVMO $=57$ contre 43 p. 100 , respectivement). De même, les feuilles de Cordyla pinnata prélevées en novembre sont plus digestibles que celles d'août (DIVMO $=44$ et 34 , respectivement).

La DIVMO des gousses de légumineuses ne semble pas liée à celle des feuilles : pour Acacia albida et Acacia tortilis, les gousses sont plus digestibles que les feuilles (55 
contre 42 p. 100 et 68 contre 44 p. 100 , respectivement) tandis que pour Acacia nilotica (51 contre 69 p. 100) on observe le contraire.

Pour les feuilles, fleurs et fruits des autres familles, les DIVMO sont très variables d'une espèce à une autre sans qu'il soit possible, en l'état actuel des résultats, d'attribuer les différences à un genre ou à une famille. Par exemple, les feuilles de Combretum aculeatum, très appétées, ont une DIVMO moyenne plus élevée que celle de Combretum nigricans $(58$ contre 25 p. 100). De même, Heeria insignis et Sclerocarya birrea, bien que de la même famille, ont des feuilles de digestibilité très différente (24 et 48 p. 100, respectivement). Parmi les échantillons étudiés, certains sont très digestibles ; on retiendra en particulier les feuilles et fleurs de Calotropis procera, celles de Balanites aegyptiaca, Combretum aculeatum, Maerua angolensis, Securinega virosa et Securidaca longepedunculata.

\section{Dégradation et dégradabilité le rumen}

Les taux de dégradation de la matière sèche et des matières azotées des échantillons aux différents temps d'incubation et les paramètres du modèle d'ORSKOV (15) sont donnés aux tableaux II et III et aux figures 1 à 7 .

La dégradabilité théorique de la matière sèche (DTMS) et celle des matières azotées (DTMA) ont des variations parallèles, de 34 à 78 p. 100 et de 24 à 86 p. 100 , avec des valeurs moyennes respectives de 57 et 59 p. 100 . Les échantillons peuvent être classés comme précédemment et les espèces ayant les meilleures DIVMO sont aussi celles qui sont les plus dégradables in situ, mais il n'est pas possible de pousser plus loin la classification.

Pour une DTMS élevée (de l'ordre de 70 p. 100), on peut avoir des vitesses de dégradation comprises entre 6 et 11 p. 100 par heure. En revanche, une DTMS faible (inférieure à $50 \mathrm{p}$. 100) correspond toujours à des vitesses de dégradation faibles de l'ordre de 2 à 5 p. 100.

Les DTMA sont étroitement liées aux DTMS $(r=0,68)$ mais pas aux teneurs en matières azotées (relation non significative, $r=0,09$ ). Pour faciliter la compréhension de l'exposé, seuls les résultats relatifs à la dégradation des matières azotées seront commentés. En effet, l'utilisation digestive de l'azote semble être le critère le plus important pour classer les ligneux suivant leur valeur nutritive pour le cheptel sahélien en saison sèche. L'examen des valeurs extrêmes prises par la DTMA met en évidence une très faible disponibilité de l'azote d'Anogeissus leio- carpus (DTMA = 24 p. 100) et celles très élevés de Bombax costatum (DTMA $=86$ p. 100) et de Boscia senegalensis (DTMA = 85 p. 100).

Les matières azotées des échantillons de Asclepiadaceae, Bombacaceae, Capparidaceae et Polygalaceae ont été les plus dégradées (DTMA supérieure à 70 p. 100 , et $c$ proche de 8 p. 100). Celles des légumineuses* et de Tiliaceae ont eu un profil intermédiaire (DTMA comprise entre 50 et 70 p. 100). Enfin, l'azote des Anacardiaceae et des Combretaceae a le plus souvent été faiblement dégradé (DTMA proche de 40 p. 100, à l'exception de C. glutinosum ${ }^{\star \star}$ ).

Pour les familles les plus représentées, il est possible de comparer les résultats entre espèces et parfois même entre organes.

Chez les Mimosaceae, les matières azotées des gousses d'A. albida, $A$. adansonii et $A$. tortilis ainsi que les feuilles d'A. senegal ont les vitesses de dégradation les plus élevées $(6,6,7$ et 11 p. 100 , respectivement). Inversement, les feuilles d'A. albida, celles d' $A$. ataxacantha, $A$. sieberiana et $A$. seyal ont la DTMA et la vitesse de dégradation de l'azote les plus faibles (c et DT varient, respectivement, de 1,3 à 2,5 et de 34 à 48 p. 100). Les autres échantillons de Mimosaceae (gousses d' $A$. raddiana, feuilles d' $A$. albida) occupent une position intermédiaire. Parmi les Combretaceae, l'échantillon de $C$. glutinosum a la DTMA la plus élevée (72 p. 100), ceux de $C$. nigricans et $A$. leiocarpus (DTMA $=46$ et 24 p. 100 respectivement) sont peu dégradables en dépit de leurs teneurs en MAT élevées (140 et $195 \mathrm{~g} / \mathrm{kg}$ de MS, respectivement).

Certaines espèces n'appartenant pas aux familles des légumineuses et des Combretaceae se distinguent par une teneur en MAT comprise entre 120 et $230 \mathrm{~g} / \mathrm{kg}$ de MS, une dégradation immédiate de cet azote (a) allant de 40 à 85 p. 100, une vitesse de dégradation comprise entre 4 et 11 p. 100 , et, finalement, une dégradation après $72 \mathrm{~h}$ de l'ordre de 80 p. 100. Il s'agit de Bombax costatum et Adansonia digitata (Bombacaceae), Calotropis procera (Asclepiadacee), Grewia bicolor (Tiliaceae) et Boscia senegalensis (Capparidaceae). On les considère provisoirement comme les espèces offrant le plus d'intérêt, parmi celles étudiées, pour la supplémentation azotée du cheptel en saison sèche. Des essais in vivo sont en cours pour mieux préciser leur valeur alimentaire.

\footnotetext{
* Exceptions : Acacia sieberiana, A. seyal (NDLR).

** Combretum aculeatum n'a pas été étudié par la méthode in situ (NDLR).
} 


\section{S. T. Fall}

TABLEAU III Dégradation in situ de l'azote des ligneux. Premiers résultats.

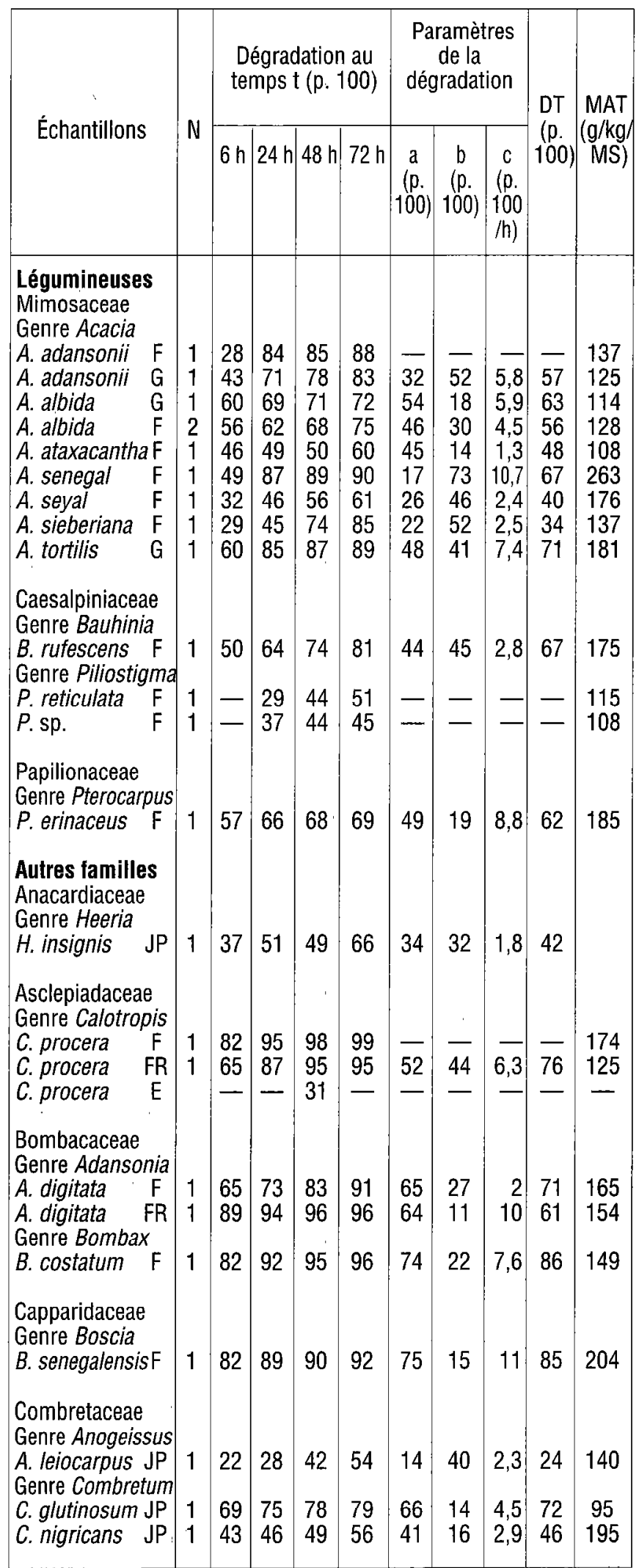

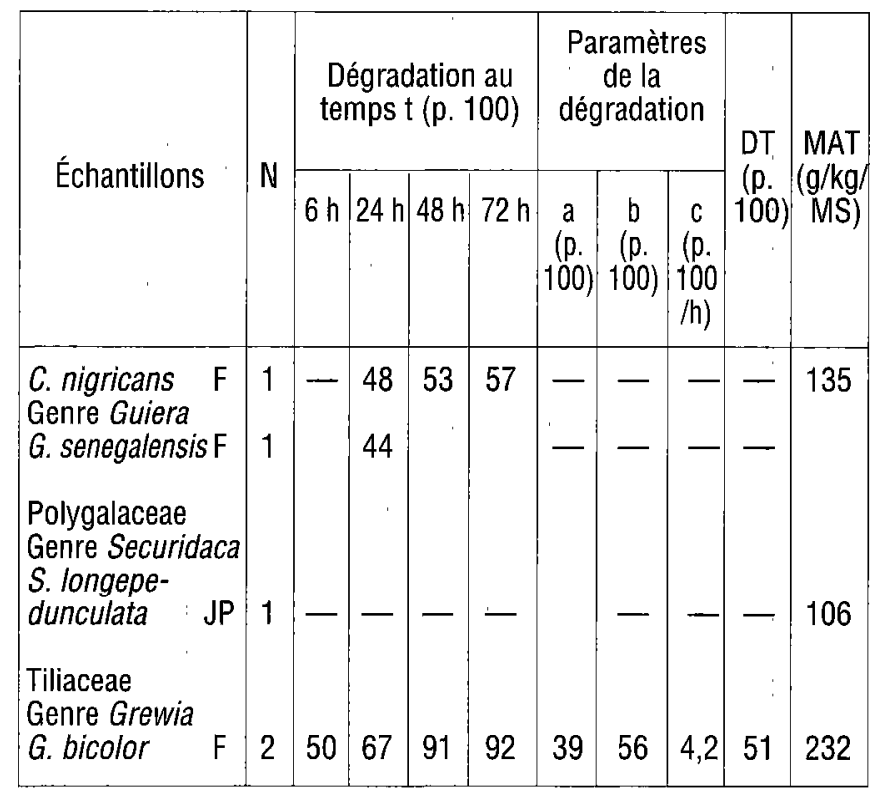

$F$ : feuilles ; $F L$ : fleurs ; $F R$ : fruits ; $G$ : gousses de légumineuses ; $E$ : écorces; JP ; jeunes pousses (= jeunes rameaux, feuilles). 
Dégradation (P. 100)

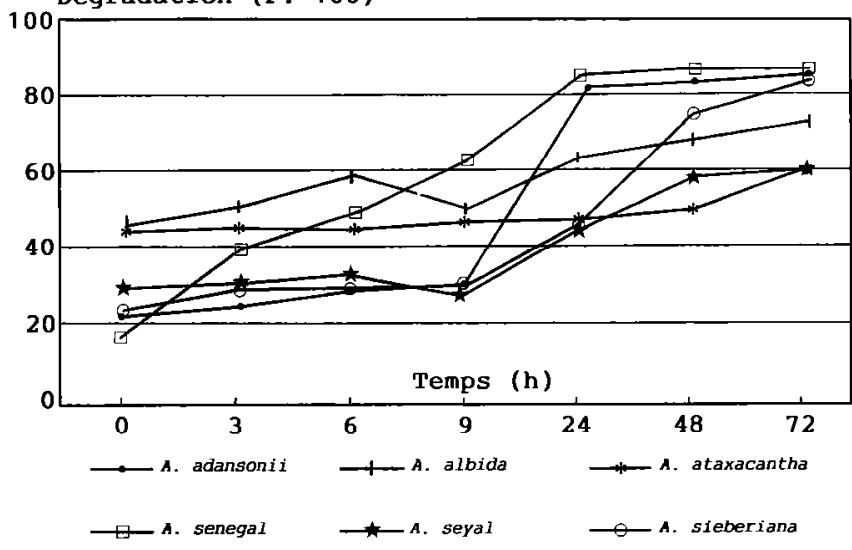

Fig. 1 : Cinétique de dégradation dans le rumen des matières azotées totales (MAT) de ligneux fourragers mesurée par la méthode in situ. feuilles de Mimosaceae.

Déqradation (P. 100)

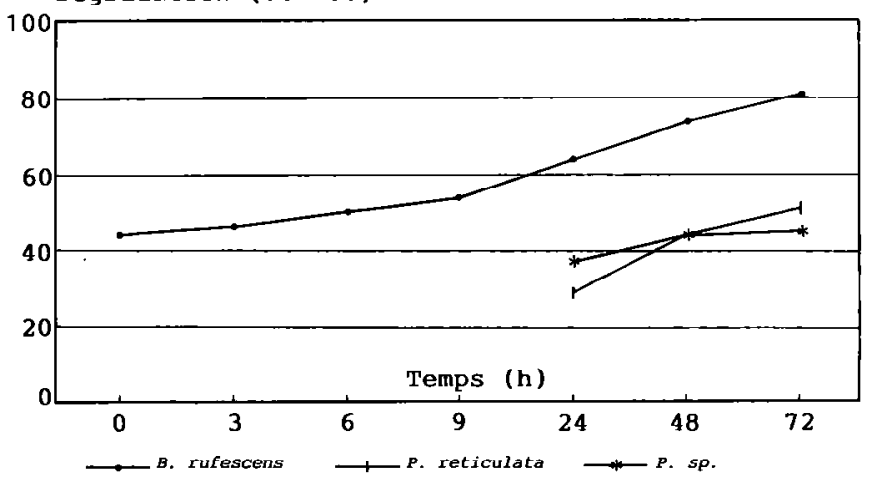

Fig. 3 : Cinétique de dégradation dans le rumen des matières azotées totales (MAT) de ligneux fourragers mesurée par la méthode in situ feuilles de Caesalpiaceae.

Dégradation (P. 100)

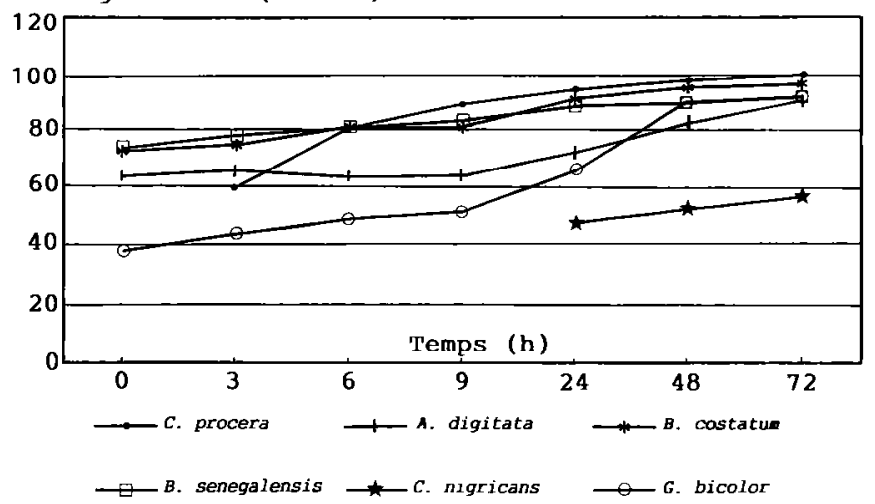

Fig. 5 : Cinétique de dégradation dans le rumen des matières azotées totales (MAT) de ligneux fourragers mesurée par la méthode in situ feuilles d'autres familles.

\section{Dégradation (P. 100)}

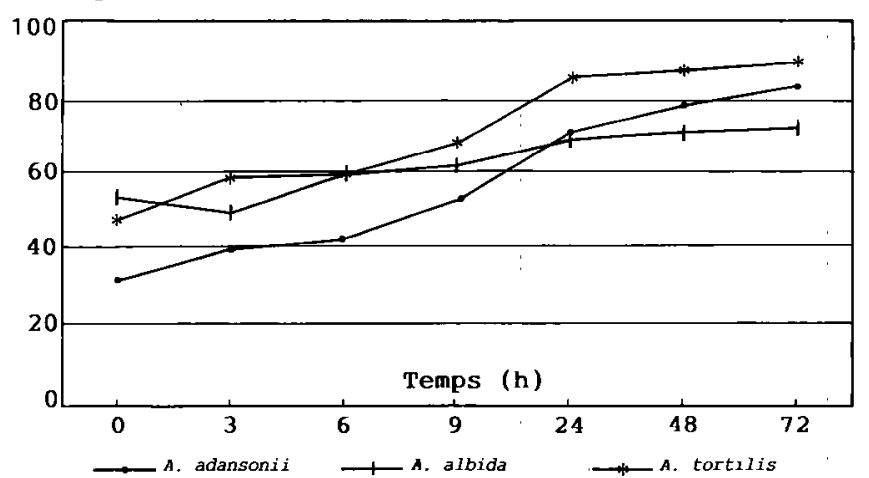

Fig. 2 : Cinétique de dégradation dans le rumen des matières azotées totales (MAT) de ligneux fourragers mesurée par la méthode in situ : gousses de Mimosaceae.

Dégradation (P. 100)

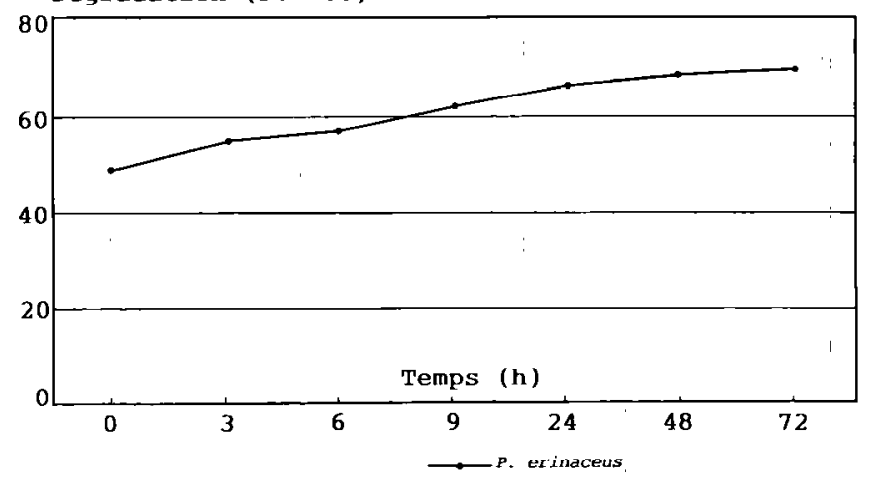

Fig. 4 Cinétique de dégradation dans le rumen des matières azotées totales (MAT) de ligneux fourragers mesurée par la méthode in situ : feuilles de Papilionaceae.

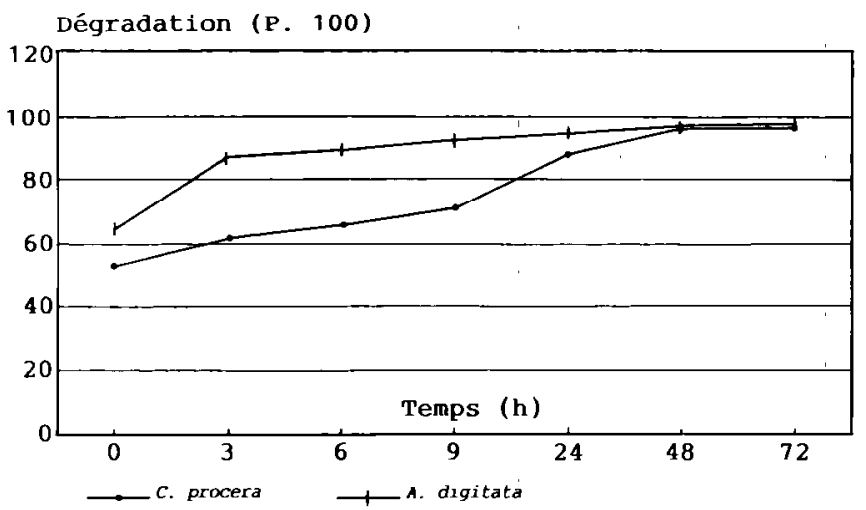

Fig. 6 Cinétique de dégradation dans le rumen des matières azotées totales (MAT) de ligneux fourragers mesurée par la méthode in situ : fruits d autres familles 
Dégradation (P. 100)

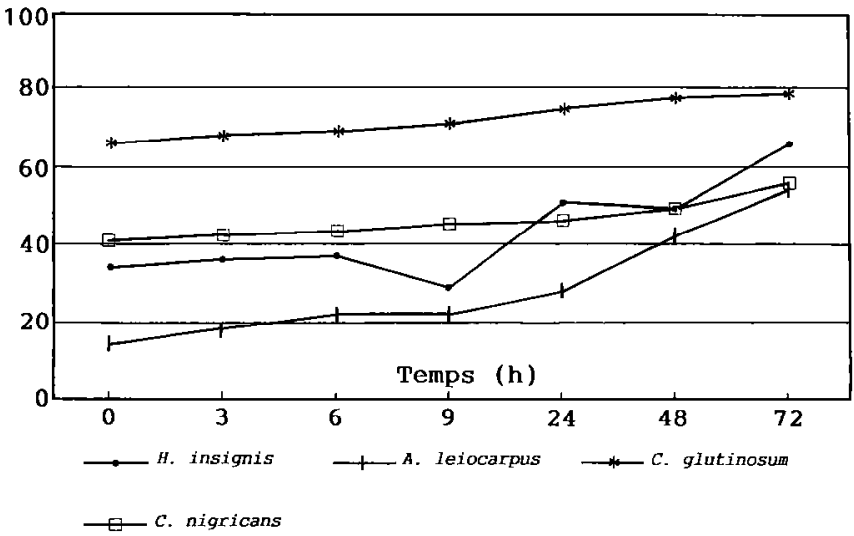

Fig. 7 : Cinétique de dégradation dans le rumen des matières azotées totales (MAT) de ligneux fourragers mesurée par la méthode in situ : jeunes pousses d'autres familles.

\section{Relations entre dégradabilité théorique et dégradation}

La dégradabilité théorique (DT) est bien expliquée par la dégradation mesurée à $24 \mathrm{~h}$ d'incubation $(P<0,01)$, laquelle semble être un bon indicateur de la dégradabilité des ligneux (tabl. IV) :

$y 1=0,78 \times 1+5,3(n=28 r=0,96, E T R=3,3)$,

$y 2=0,79 \times 2+5,4(n=28, r=0,89, E T R=7,4)$,

avec $y 1=$ DTMS, $x 1=$ dégradation de la matière sèche à 24 h d'incubation, y2 = DTMA, $x 2$ = dégradation des MAT à $24 \mathrm{~h}$ d'incubation.

Une confirmation de ces équations sur un nombre plus important d'observations simplifierait l'étude de la cinétique de dégradation in situ des ligneux.

Une corrélation significative est observée $(P<0,01)$ entre DIVMS et dégradation in situ à 9,24 et 48 h d'incubation. Pour 14,18 et 17 observations, $r$ a été, respectivement, de $0,57,0,75$ et 0,84 et l'erreur standard de 14,11 et 10 p. 100 .

TABLEAU IV Coefficients de corrélation entre dégradation au temps t et dégradabilité.

\begin{tabular}{|c|c|c|c|c|c|c|}
\hline \multirow{2}{*}{ DT } & \multicolumn{6}{|c|}{ Temps d'incubation (h) } \\
\cline { 2 - 7 } & 3 & 6 & 9 & 24 & 48 & 72 \\
\hline DTMS & 0,85 & 0,93 & 0,95 & 0,96 & 0,91 & 0,86 \\
\hline DTMA & 0,82 & 0,85 & 0,88 & 0,89 & 0,76 & 0,70 \\
\hline
\end{tabular}

\section{DISCUSSION}

Dans la littérature, les données concernant la digestion in vivo, in vitro ou in situ des ligneux des zones tropicales sont rares, contrairement aux résultats d'analyses chimiques qui sont nombreux mais peu utilisables. En effet, la cellulose brute et les MAT ont une faible signification pour ce type d'aliments riches en lignine, en tannin et en d'autres facteurs antinutritionnels. L'accent doit être mis, actuellement, sur l'examen des fractions pariétales (hémicellulose, cellulose, lignine) et azotées (azote soluble dégradable lié aux parois indigestibles...), sur les méthodes enzymatiques (cellulases, protéases...) et biologiques (digestibilité in vivo, in vitro et in situ) de mesure de leur disponibilité digestive, et sur la mesure des performances chez l'animal.

Les variations liées aux stades de récolte, aux conditions d'échantillonnage et de conservation, aux techniques d'analyse ne permettent pas de procéder à des comparaisons rigoureuses et détaillées des résultats : il est cependant possible de mettre en parallèle les classifications d'espèces obtenues par les différents auteurs $(2,10,11)$.

En ce qui concerne les feuilles d'Acacia albida, une DMS moyenne (53 p. 100) proche de nos résultats a été rapportée par DICKO en 1979, cité par le HOUÉROU (13), avec la méthode in vivo. Cependant, l'écart assez important (14 points) observé au cours de l'étude entre le début et la fin du cycle (janvier-août) montre une nette influence du stade phénologique. Pour les gousses, la DIVMS a été stable, aucune variation d'ordre phénologique n'a été observée. Les résultats ont été supérieurs à ceux de DICKO, mais, les dates de récolte n'ayant pas été mentionnées, il est difficile d'expliquer ces différences. En ce qui concerne les gousses de $B$. rufescens, des résultats supérieurs ( 51 contre $39 \mathrm{p}$. 100) ont été obtenus par le même auteur. L'état actuel de nos résultats ne permet pas d'interpréter ces différences ainsi que les variations de digestibilité de Securinega virosa (échantillons récoltés à la même période) et celles de Balanites aegyptiaca (entre les récoltes de juillet et novembre). La place qu'occupent ces espèces dans les écosystèmes pastoraux sahéliens justifie la poursuite des analyses pour préciser la cause de ces variations.

Les DIVMS et DIVMO ont une évolution parallèle. La différence plus importante entre DIVMS et DIVMO observée chez les ligneux par rapport aux fourrages classiques doit résulter du fait que les ligneux sont probablement plus riches en minéraux solubles dans le mélange salive artificielle et jus de rumen.

La dégradabilité de la matière sèche est un critère assez intéressant pour apprécier, à appétibilité égale, l'ingestibilité des aliments. En effet, la quantité ingérée est en étroite liaison avec la vitesse de dégradation de la matière sèche dans le rumen. II faudrait cependant associer cette dernière avec la présence éventuelle de substances, cer- 
taines pouvant être toxiques* et antinutritionnelles, qui peuvent limiter l'appétibilité des espèces ligneuses et, de ce fait, leur ingestion. Ainsi, les feuilles et gousses de légumineuses, les feuilles de Calotropis procera, Guiera senegalensis et Adansonia digitata distribuées aux ovins aux taux de 10 à 50 p. 100 de la ration sont totalement consommées, même si les animaux ne les acceptent qu'après 15 jours d'adaptation. Ceci n'est pas le cas pour Boscia senegalensis, espèce pourtant très dégradable, mais dont les jeunes pousses sont quasiment rejetées alors que les feuilles âgées sont acceptées par les moutons (FALL, non publié). Une substance, peut-être antinutritionnelle, à teneur ou à forme variable en fonction de l'âge, est à rechercher pour expliquer ces variations de consommation.

En ce qui concerne la dégradation des matières azotées, les résultats obtenus à $48 \mathrm{~h}$ d'incubation ont été comparables à ceux de KONÉ (10) pour les feuilles d'A. adansonii et de $P$. reticulatum, ainsi que les jeunes pousses d' $A$. albida et de $B$. rufescens. Ils sont en revanche différents pour les feuilles d' $A$. seyal, $A$. ataxacantha, $A$. albida et $C$. nigricans (tabl. $V$ ). Ces différences pourraient s'expliquer par des facteurs de variation déjà cités, mais aussi par des conditions d'expérimentation non identiques (échantillons, espèces animales, race, régime alimentaire, tissu de nylon, environnement). WOODWARD et REED (20) signalent une bonne digestibilité de l'azote des feuilles d'A. adansonii, avec des valeurs peu différentes des nôtres (85 contre 92 p. 100). Ils mentionnent aussi une haute teneur en substances phénoliques.

TABLEAU V Dégradabilité des MAT à 48 h d'incubation. Comparaison de résultats (p. 100).

\begin{tabular}{|c|c|c|c|}
\hline Espèces & Organes & $\begin{array}{c}\text { Source } \\
\text { Koné } \\
(1987)\end{array}$ & $\begin{array}{c}\text { Source } \\
\text { Fall } \\
(1989)\end{array}$ \\
\hline Acacia adansonii & Feuilles & 75 & 85 \\
Acacia albida & Jeunes pousses & 91 & 86 \\
Acacia albida & Feuilles & 20 & 53 \\
Acacia ataxacantha & Feuilles & 22 & 50 \\
Acacia seyal & Feuilles & 82 & 56 \\
Boscia senegalensis & Fruits & 92 & -90 \\
Boscia senegalensis & Feuilles & - & 90 \\
Bauhinia rufescens & Jeunes pousses & $83-75$ & 74 \\
Combretum nigricans & Feuilles & 66 & $53-50$ \\
Piliostigma reticulata & Feuilles & 34 & $35-53$ \\
\hline
\end{tabular}

\footnotetext{
* Il n'y a pas que des substances toxiques qui peuvent limiter l'appétibilité.
}

Les amplitudes de variation de la vitesse de dégradation (c) et de la dégradation immédiate (a) des MAT ont été très grandes. Elles constituent des critères de classification importants car elles déterminent la disponibilité des nutriments dans les pré-estomacs. En effet, certain'es espèces à dégradation rapide comme les genres Boscia ou Calotropis devraient engendrer une élévation rapide du taux d'ammoniac dans le rumen; elles seront donc plus indiquées pour la supplémentation des fourrages pauvres en azote.

La non-disponibilité des MAT des feuilles d'A. ataxacantha, $A$. sieberiana et $A$. seyal est explicable par une teneur en lignine très élevée (10) et surtout par un fort pourcentage d'azote bloqué dans l'ADF (NADF), en particulier pour $A$. seyal, dont DIAGAYETE (2) signale un taux de NADF de 11 p. 100 de l'azote total, supérieur à la moyenne ( 6 p. 100) des espèces qu'il a étudiées. Cependant, WOODWARD et REED (20) rapportent une bonne utilisation digestive de l'azote pour cette espèce. Pour DIAGAYETE (2), des teneurs élevées en tannins seraient responsables de la faible dégradabilité des feuilles d'A. albida.

Les résultats mettent en évidence des variations irrégulières, quelquefois non explicables. Si la famille, l'espèce et l'organe peuvent être des critères de variation évidents, les variations phénologiques sont difficiles à analyser. En effet, la plupart des espèces décrites ont un cycle végétatif variable en fonction du site et de l'année. La température ne semble pas avoir un effet aussi important que la pluviométrie et sa répartition, ainsi que l'hygrométrie, la photopériode et le mode d'exploitation, qui influent fortement sur la croissance et le cycle des ligneux (6). Pour contourner ces variations, les récoltes d'échantillons doivent être accompagnées de commémoratifs complets décrivant l'organe, son stade de développement, le site et la date de prélèvement ainsi que le mode de conservation.

\section{CONCLUSION}

L'étude de l'utilisation digestive des ligneux fourragers par les ruminants a mis en évidence une grande dispersion des résultats selon les espèces, l'âge et l'organe. 'Si les ligneux peuvent être riches en énergie brute et en matières azotées totales, leur utilisation digestive peut être médiocre à certains stades de développement et pour certains organes.

Un plus grand nombre d'analyses est nécessaire pour préciser ces variations et améliorer l'identification des espèces les plus performantes. Notons cependant une concordance des résultats in vitro et in situ quant à la bonne digestibilité d'A. nilotica, $A$. seyal et $A$. tortilis (Mimosaceae), Calotropis procera (Asclepiadaceae), Securicada longepedunculata (Polygalaceae), Boscia senegalensis (Capparidaceae), Adansonia digitata et Bombax costatum (Bombacaceae). 
FALL (S.T.). In vitro digestibility and in situ rumen degradability of browse plants from natural pastures in Senegal. First results. Revue Elev. Méd. vét. Pays trop., 1991, 44 (3) : 345-354

To evaluate the apparent digestibility of trees and shrubs browsed in Sahelian and Sudanian areas of Senegal, an in vitro method was used (Tilley and Terry's method : dry matter (IVDM) and organic matter (IVOM) digestibility) as well as an in situ method (intra-ruminal degradability of dry matter and nitrogen). In 58 analysed samples, the average IVDM and IVOM were 51 and $42 \%$, ranging from 26 to $88 \%$ and from 15 to $84 \%$, respectively. A total of 38 degradation profiles (in sacco) involving 24 species gave a mean degradability of 59 and $57 \%$ for nitrogen and dry matter, respectively. Variation factors linked to species, age and plant parts were observed and a provisional classification of studied species proposed. Key words : Browse plant - Fodder - Ruminant - In vitro digestibility - In situ dcgradability Senegal.
FALL (S.T.). Digestibilidad in vitro y degradabilidad in situ en la panza de forrajes leñosos disponibles en pastos naturales en Senegal. Primeros resultados. Revue Élev. Méd. vét. Pays trop., 1991, 44 (3) : 345-354

Para evidenciar la utilización digestiva de los forrajes leñosos disponibles en pastos naturales de las zonas sudanesa y saheliana del Senegal, se determinaron la digestibilidad in vitro (método de Tilley-Terry) y la degradabilidad en la panza (in situ). De 58 muestras analizadas in vitro, la digestibilidad in vitro de la materia seca es de $\mathbf{5 1}$ por termino medio con extremos de 88 a $26 \mathrm{p}$. 100. La digestibilidad in vitro de la materia orgánica varió de 84 a 15 con un promedio de 42 p. 100. Se realizaron 38 perfiles de degradación en la panza en 24 especies. Son respectivamente de 59 y 57 p. 100 la degradabilidad media de proteinas brutas y la de la materia seca. Se observaron factores de variación según la especie, el órgano y la edad. Se propone una jerarquía provisional de las especies estudiadas. Palabras claves : Planta leñosa Forraje Rumiante Digestibilidad in vitro - Degradabilidad in situ - Senegal.

\section{BIBLIOGRAPHIE}

1. RIPF.A. Recueil des méthodes d'analyse des Communautés européennes. Paris, BIPEA, 1976.

2. DIAGAYETE (M.). Untersuchungen zur Eiweiterung der Kenntnisse über den futterwert West Africa mischer Futterpflanzen. Diploomagraringenieur, Univ. Hohenheim, 1981.

3. DOREAU (B.M.), VERITE (R.), CHAPOUTOT (P.). Méthodologie de mesure de la dégradabilité in sacco de l'azote des aliments dans le rumen. Bull. tech. Cent. Rech. Zoot. vét. Theix, 1987: 5-7.

4. FALL (S.T.). Utilisation digestive par les ruminants domestiques de ligncux fourragers disponibles au Sénégal. Méthodologie et premiers résultats. Dakar, ISRA-LNERV, 1988.89 p. (Alim. Nutr. $\mathrm{n}^{\circ} 59$ ).

5. FALL (S.T.). Utilisation d'Acacia albida et de Calotropis procera pour améliorer la ration des petits ruminants au Sénégal, In : WILSON (R.T.), MELAKU (A.), eds. African small ruminant research and development. Addis Abeba, ILCA, 1989. P. 155-167.

6. GROUZIS (M.), SICOT (M.). A method for the phenological study of browse population in the Sahel : the influence of some ecological factors. In : LE HOUEROU (H.N.), ed. Browse in Africa : the current stage of knowledge. Addis Ababa, II CA, 1980. P. 233-240.

7. GUERIN (H.). Alimentation des ruminants domestiques sur pâturages naturels sahéliens et sahélo-soudaniens. Étude méthodologique dans la région du Ferlo au Sénégal. Thèse doct.-ing. agronome, Montpellier, ENSAM, 1987.211 p.

8. GUERIN (H.), FRIOT (D.), M'BAYE (N.), RICHARD (D.), DIENG (A.). Régime alimentaire des ruminants domestiques (bovins, ovins, caprins) exploitant les parcours naturels sahéliens et soudano-sahéliens. II. Essais de description du régime par l'étude du comportement alimentaire. Facteurs de variation des choix alimentaires et conséquences nutritionnelles. Revue Elev. Méd. vét. Pays trop., 1988, 41 (4) : 427-440.

9. HARRINGTON (G.), WILSON (A.D.). Method of measuring secondary production from browse. In : LE HOUÉROU (H.N.), ed. Browse in Africa : the current stage of knowledge. Addis-Abeba, ILCA, 1980. P. 255-260.

10. KONÉ (A.R.), GUERIN (H.), RICHARD (D.). Contribution à la mise au point d'une méthode d'étude de la valeur nutritive des fourrages ligneux. Maisons-Alfort, IEMVT, 1989. P. $789-809$ (Études et synthèses, $n^{\circ}$ 30).

11. KONÉ (A.R.), RICHARD (D.), GUERIN (H.). Teneur en constituants pariétaux et en matières azotées de ligneux fourragers d'Afrique occidentale. In : XVI' congrès international des herbages, 4-11 octobre 1989, Nice, France. Versailles, Association française pour la production fourragère, 1989. Vol. II, p. 947-948.

12. LAMPREY (H.F.), HERLOCKER (D.J.), FIELD (C.R.). Report on the stage of knowledge of browse in West Africa. In : LE HOUÉROU (H.N.), ed. Browse in Africa : the current stage of knowledge. Addis Abeba, ILCA, 1980. P. 33-54.

13. LE HOUÉROU (H.N.). Chemical composition and nutritive value of browse in tropical West Africa. In : LE HOUÉROU (H.N.), ed. Browse in Africa : the current stage of knowledge. Addis Ababa, ILCA, 1980. P. 261-289.

14. McLLOD. Plant tannins. Their rolc on foragc quality. Nutr. Abstr. Rev., 1974, 14 (11) : 803815.

15. ORSKOV (E.R.). The use of nylon bag technique for the evaluation of feedstuffs. Trop. Anim. Prod., 1980 (5) : 195-213.

16. REED (J.D.), HOVARTH (P.J.), ALLEN (M.S.), VAN SOEST (P.J.). Gravimetric determination of soluble phenolics including tannins from leaves by precipitation with trivalent ytterbium. J. Sci. Fd Agric., 1985, 36:255-261.

17. RIVIERE (R.). Manuel d'alimentation des ruminants domestiques en milieu tropical. Paris, Ministère de la Coopération, 1978. P. 472-481 (Manuels et précis d'élevage, $n^{\circ} 9$ ).

18. STAT-ITCF. Programme informatique rédigé par les ingénieurs. Paris, ITCF.

19. TILLEY (J.M.A.), TERRY (R.A.). A two-stage technique for the in vitro digestion of forage crops. J. Br. Grassld Soc., 1963 (18) : 104-107.

20. WOODWARD (A.), REED (J.D.). Influence des substances polyphénoliques sur la valeur nutritive des fourrages ligneux. Synthèse des recherches menées par le CIPEA. Bull CIPEA, 1989, 35:2-13. 